\title{
INDEX OF VOLUME XXV.
}

Archibald, R. C. Note on Editions of von Staudt's Geometrie der Lage, 132.

- See Reviews, under Smith, D. E.

Bateman, H. Rotating Cylinders and Rectilinear Vortices, 358.

BeLl, E. T. A Partial Isomorph of Trigonometry, 311.

Bennett, A. A. Products of Skew-Symmetric Matrices, 455.

Bernstein, B. A. Reports of Meetings of the San Francisco Section: December Meeting, 193; April Meeting, 393.

Birkнoff, G. D. The Scientific Work of Maxime Bôcher, 197.

Blichreldt, H. F. Report on the Theory of the Geometry of Numbers, 449.

BuIss, G. A. Solutions of Differential Equations as Functions of the Constants of Integration, 15.

Blumberg, H. A Theorem on Linear Point Sets, 350.

Bôcher, M. See Birkhoff, G. D., and Osgood, W. F.

Brown, E. W. See Reviews, under Annuaire.

Carmichael, R. D. General Aspects of the Theory of Summable Series, 97.

— See Reviews, under Borel, Macaulay, Macrobert, Parker, Picard, Smith, C. M.

Chittenden, E. W. On the Heine-Borel Property in the Theory of Abstract Sets, 60.

Cole, F. N. Reports of Meetings of the American Mathematical Society: Twenty-Fifth Summer Meeting, 49; October Meeting, 145; April Meeting, 433.

Craig, C. F. See Reviews, under Maurus.

Cummings, L. D. The Trains for the 36 Groupless Triad Systems on 15 Elements, 321.

Curtis, M. F. On the Rectifiability of a Twisted Cubic, 87.

Daniell, P. J. Integrals around General Boundaries, 65.

— A General Form of Green's Theorem, 353.

The Derivative of a Functional, 414.

Davis, E. W. See Hedrick, E. R.

Dickson, L. E. Mathematics in War Perspective, 289.

- Applications of the Geometry of Numbers to Algebraic Numbers, 453.

Dowling, L. W. See Reviews, under Fazzari, Scritti matematici.

Dresden, A. See Reviews, under Shaw.

EmcH, A. On Plane Algebraic Curves with a Given System of Foci, 157. 
- On a certain Generation of Rational Circular and Isotropic Curves, 397.

- See Reviews, under de Montessus de Ballore.

Evans, G. C. Corrections and Note to the Cambridge Colloquium of September, 1916, 461.

Fond, W. B. A Conspectus of the Modern Theory of Divergent Series, 1.

Graves, G. H. See Reviews, under Thompson.

Hallett, G. H. Concerning the Definition of a Simple Continuous Arc, 325.

Hancock, H. On the Evaluation of the Elliptic Transcendents $\eta_{2}$ and $\eta_{2}^{\prime}, 150$.

Hayasht, T. On the Problem of the Resistance Integral, 131.

A Theorem on Areas, 324.

Hedrick, E. R. In Memoriam: Ellery William Davis, 36.

Longley, W. R. See Reviews, under Lecornu.

Lovits, W. V. See Reviews, under Secrist.

McAtee, J. E. The Transformation of a Regular Group into its Conjoint, 326.

Miller, G. A. Determinant Groups, 69.

- Groups Containing a Relatively Large Number of Operators of Order Two, 408.

Moore, C. L. E. Translation Surfaces in Hyperspace, 75.

Moore, C. N. Applications of the Theory of Summability to Developments in Orthogonal Functions, 258.

- See Reviews, under Karpinski, Pringsheim.

Moore, R. L. Continuous Sets that have no Continuous Sets of Condensation, 174.

Morgan, F. M. See Reviews, under Carey, Long, Williams.

Moulton, E. J. Reports of Meetings of the American Mathematical Society: Twenty-Fifth Annual Meeting, 241; March Meeting at Chicago, 385.

OsGood, W. F. On a Theorem of Oscillation, 216.

- The Life and Services of Maxime Bôcher, 337.

Pitcher, A. D. See Reviews, under Young.

Ponter, M. B. Derivativeless Continuous Functions, 176.

Rowe, J. E. Related Invariants of Two Rational Sextics, 34.

Shaw, J.B. See Reviews, under Perry.

Sommerville, D. M. Y. Quadratic Systems of Circles in Non-Euclidean Geometry, 161.

Vandiver, H. S. Proof of a Property of the Norm of a Cyclotomic Integer, 221 . 
- On the First Factor of the Class Number of a Cyclotomic Field, 458. Wear, L. E. The Self-Dual Plane Rational Quintic, 405.

Weaver, J. H. Some Algebraic Curves, 85, 329.

White, H. S. See Reviews, under Coolidge.

Whittemore, J. K. Trajectories and Flat Points on Ruled Surfaces, 223.

WINGER, R. M. Involutions on the Rational Cubic, 27.

\section{Reviews.}

Annuaire du Bureau des Longitudes pour l'An 1919, E. W. Brown, 473.

Benedict, H. Y. See Karpinski.

Borel, ? E. Leçons sur les Fonctions monogènes d'une Variable complexe, rédigées par G. Julia, R. D. Carmichael, 230.

Brenke, W. C. See Long, E.

Calhoun, J. W. See Karpinski.

Carey, F. S. Infinitesimal Calculus, F. M. Morgan, 472.

Coolidge, J. L. A Treatise on the Circle and the Sphere, H. S. Whiтe, 464.

Fazzari, G. I Numeri reali e l'Equazione esponenziale $a^{x}=b$ per le Scuole Medie superiori, L. W. Dowling, 470.

Gerbaldi, F. See Scritti matematici.

Hedrick,'E. R. See Smith, C. M.

Julia, G. See Borel, E.

Karpinski, L. C., Benedict, H. Y., and Calhoun, J. W. Unified Mathematics, C. N. Moore, 467.

Lecornu, L. Cours de Mécanique, volume 3, W. R. Longley, 90.

Long, E., and Brenke, W. C. Plane Geometry, F. M. Mongan, 422.

Loria, G. See Scritti matematici.

Macaulay, F. S. The Algebraic Theory of Modular Systems, R. D. Carmichael, 276.

Macrobert, T. M. Functions of a Complex Variable,IR. D. CARMichaed, 377.

Maurus, E. J. An Elementary Course in Differential Equations, C. F. Craig, 89.

de Montessus de Ballore, R. Leçons sur les Fonctions elliptiques en Vue de leurs Applications, A. EMCH, 378.

Morgan, F. M. See Young, J. W.

d'Ovidio, E. See Scritti matematici.

Parker, G. W. Elements of Optics for the Use of Schools and Colleges, R. D. Carmichael, 234.

Perry, H. Theories of Energy, J. B. Shaw, 423.

Picard, E. Les Sciences mathématiques en France depuis un DemiSiècle, R. D. Carmichael, 180. 
Pringsheim, A. Vorlesungen über Zahlen- und Funktionenlehre. Erster Band, erste Abtheilung, C. N. Moore, 470.

Scritti matematici offerti ad Enrico d'Ovidio in Occasione del suo LXXV Genetliaco, 11 Agosto 1918, e publicati per cura di F. Gerbaldi e G. Loria, L. W. Dowling, 417.

Secrist, H. An Introduction to Statistical Methods, W. V. Lovitr, 89.

Seely, C. E. See Smith, D. E.

Shaw, J. B. Lectures on the Philosophy of Mathematics, A .Dresden, 374.

Smith, C. M. Electric and Magnetic Measurements, edited by E. R. Hedrick, R. D. Carmichael, 91.

Smith, D. E. and Seely, C. E. Union List of Mathematical Periodicals, R. C. Archibald, 134.

Thompson, A. W. H. A New Analysis of Plane Geometry, Finite and Differential, G. H. Graves, 89.

Williams, J. H. and Williams, K. P. Plane Geometry, F. M. Morgan, 423. Williams, K. P. See Williams, J. H.

Young, J. W. and Morgan, F. M. Elementary Mathematical Analysis, A. D. Pitcher, 185.

Corrections, 38, 329.

Index of Volume XXV, 492.

New Publications, 46, 94, 142, 190, 238, 285, 333, 381, 429, 480.

Notes, 39, 92, 137, 188, 235, 279, 329, 379, 424, 474.

Papers Read before the Society and Subsequently Published, TwentyEighth Annual List of, 484 .

\section{Notes AND Other Items.}

Academies, Associations, Congresses, and Societies:

American Mathematical Society: Annual Meeting, 92, 137, 145, 235; April Meeting, 279; Chicago Section, 279; Colloquium, 474; Election of Officers, 242; February Meeting, 146; New Members Admitted, 50, 145, 242, 433; Presidential Address, 92, 137, 241; Southwestern Section, 92, 146; Statistics, 242, 329; Summer Meeting, 242, 385, 434, 474; Symposium, 279; Transactions, 92, 188, 279, 424, 434 .

Associations for the Advancement of Science, American, 137; British, 380.

Associations of Mathematics Teachers, Middle States and Maryland, 475; New Jersey, 475.

American Academy of Arts and Sciences, 282; Brussels Conference, 474; Circolo Matematico di Palermo, 379; Committee on Mathematical Requirements, 474; Copenhagen Mathematical Society, 380; Danish Academy of Sciences, 476; Edinburgh Mathematical Society, 39, 188, 281, 380, 425, 475; Heidelberg Academy of Sciences, 281; Indian Mathematical Society, 425; International Mathematical Union, 474; Italian Society of Sciences, 331; London Mathematical Society, 280, 330, 380, 475; Mathematical Association of America, 92, 137, 235, 237, 
280, 474; National Academy of Sciences, 424; Paris Academy of Sciences, 42, 139, 237; Prince Jablonowski Society, 281; Royal Society of London, 282; U. S. Ordnance Department, 474.

Bôcher Memorial, 145, 282.

Books, Announcemient of New, 43, 188.

Catalogues of Books, 94, 480.

Doctorates in Mathematics, American, 138.

Encyklopädie der mathematischen Wissenschaften, 281.

Journals: American Journal of Mathematics, 39, 137, 280, 379; American Mathematical Monthly, 237, 280; Annals of Mathematics, 137, 236, 330; Nyt Tydsskrift for Matematik, 380; Periodico di Matematica, 39; Proceedings of the National Academy of Sciences, 236; Rendiconti del Circolo Matematico di Palermo, 379; Tokyo SugakuButurigakkwai Kizi, 280; Transactions of the American Mathematical Society, 92, 188, 279, 424, 434.

Papers and Communications Presented to the Society, Authors:

Alger, P. L., 242.

Allardice, R. E., 393.

Allen, F. E., 386.

Baker, R. P., 244, 244.

Barnett, I. A., 243, 244.

Beal, F. W., 436.

Bell, E. T., 193, 193, 193, 193 $193,393,393$.

Bennett, A. A., 241.

Birkhoff, G. D., 51, 435, 435, 435.

Blichfeldt, H. F., 242, 386.

Bliss, G. A., 386.

Blumberg, H., 243, 386.

Buchanan, D., 51, 243.

Bussey, W. H., 386.

Cairns, W. D., 51.

Cajori, F., 193, 193, 393.

Chao, Y. R., 393.

Chittenden, E. W., 146, 386.

Coble, A. B., 386.

Cummings, L. D., 50.

Curtis, M. F., 51.

Daniell, P. J., 50, 244.

Dickson, L. E., 241, 386.

Dines, L. L., 386.

Dodd, E. L., 243.

Eisenhart, L. P., 436.

Emch, A., 244, 436.

Fischer, C. A., 436.

Fite, W. B., 434.

Ford, W. B., 243.

Forsyth, C. H., 51.

Franklin, P., 435.

Fry, T. C., 436.

Glenn, O. E., 50, 435, 435, 435.

Gould, A. B., 242.

Groat, B. F., 50, 51.

Gronwall, T. H., 244, 244, 244, 244,435 .
Hallett, G. H., Jr., 146.

Hancock, H., 243, 244.

Haskell, M. W., 193.

Haskins, C. N., 51.

Hazlett, O. C., 243.

Hoskins, L. M., 393.

Jackson, D., 435.

Johnson, W. W., 50.

Kasner, E., 146, 435

Keyser, C. J., 434.

Kline, J. R., 435.

Ladd-Franklin, C., 51.

Larew, G. A., 146.

Laves, K., 242.

Lefschetz, S., 244.

Lehmer, D. N., 193.

Lunn, A. C., 435.

McAtee, J. E., 146.

MacMillan, W. D., 242.

Manning, W. A., 393.

Mathewson, L. C., 50.

Miller, G. A., 50, 243, 386.

Moore, C. N., 243, 244.

Moore, R. L., 146, 146, 435.

Moulton, E. J., 386.

Nelson, A. L., 386.

Pitcher, A. D., 386.

Porter, A. D., 146.

Reynolds, C. N., Jr., 50.

Rietz, H. L., 242, 386.

Ritt, J. F., 435.

Robinson, L. B., 50, 435, 435.

Roe, E. D., Jr., 435, 435.

Roe, J. R., 50.

Roever, W. H., 242.

Rowe, J. E., 50.

Safford, F. H., 435.

Schweitzer, A. R., 51, 244, 244, 386, 435.

Sensenig, W., 243. 
Shaw, J. B., 244.

Silverman, L. L., 436.

Simon, W. G., 244.

Sisam, C. H., 243.

Smail, L. L., 393.

Sommerville, D. M. Y., 146.

Sperry, P., 393.

Stouffer, E. B., 243.
Taylor, J. S., 393.

Vandiver, H. S., 436.

Van Vleck, E. B., 386.

Veblen, O., 435.

White, H. S., 436.

Whittemore, J. K., 434.

Wilczynski, E. J., 243, 386, 386.

Personal Notes:

Adams, C. R., 284, 478; Alexander, J. W., II, 139; Almansi, E., 477;

Amaldi, U., 331; Archibald, R. C., 188, 237; Armstrong, L. E., 283.

Baldwin, J. W., 283; Barbarin, P., 237; Barnard, R. W., 93; Barnett, I. A., 44, 138, 479; Barnhart, C. A., 141; Barrow, D. F., 139; Barton, H., 480; Bass, E. W., 142; Bauer, G. N., 284, 429; Beal, F. W., 93; Belopslkÿ, A., 139; Bennett, A. A., 139; Betz, H., 478; Bill, E. G., 189, 478; Birkhoff, G. D., 137, 428, 474; Blichfeldt, H. F., 139, 279 ; Bliss, G. A., 139; Blumberg, H., 44; Bôcher, M., 45, 145, 280, 282; Bonnesen, T., 42; Borden, R. F., 44, 138; Bottasso, M., 333; Bowes, R., 480; Boyd, P. P., 478; Bray, H. E., 138; von Brill, A., 331; Brink, R. W., 429; Brown, B. H., 428; Buck, T., 139, 139; Bullard, J. A., 429; Burgess, R. W., 478; Burnside, W., 477; Burwell, W. R., 284.

Camp, C. C., 478; Campbell, A. D., 237, 478; Cantor, G., 45; Capron, P., 429; Carathéodory, C., 189; Carmichael, R. D., 44; Carus, P., 285; Chevalier, S., 139; Chittenden, E. W., 44, 140; Clements, G. R., 429; Coble, A. B., 44, 434; Cohen, T., 138; Colonnetti, G., 42; Coolidge, J. L., 477; Cornell, T. A., 284; Cosserat, E., 477; Cresse, G. H., 429; Curtiss, D. R., 434 .

Dalaker, H. H., 138; Daniels, A. L., 45; Daniels, F., 284; Dantzig, T., 284; Darboux, G., 39; Dickson, 92, 137, 279; Dillingham, A., 429; Dines, C. R., 139; Dines, L. L., 93; Dini, U., 284, 332; Doll, T., 284; Doolittle, C. L., 333; Douglas, R., 479; Dresden, A., 43, 140; Duncan, F., 140.

Elliott, W. W., 381; Eneström, E., 476; Eppes, J. B., 429; Eshleman, J. D., 93; Evans, G. C., 189, 478; Evans, H. B., 140.

Fano, G., 477; Fanta, E., 332; Fatou, P., 237; Ferguson, Z., 140; Fischer, C. A., 478; Foord-Kelcey, W., 282; Ford, L. R. 93, 283; Fraleigh, P. A., 140, 283, 429; Frary, H. D., 138; Fréchet, M., 282; Freda, E., 331; Frizell, A. B., 284; Frobenius, G., 189.

Gaba, M. G., 44; Galloway, J. N., 429; Garnett, W. H., 381; Gauthier-Villars, A., 45; Geilen, V., 282; Gilman, R. E., 478; Glasgow, J., 140; Goursat, E., 477; Graefe, F., 332; Graf, J. H., 141; Graustein, W. C., 43, 428; Green, G. M., 285; Grennan, E. B., 140; Grillier, V., 282; Gronwall, T. H., 139; Gross, W., 332, 477; Grossmann, A., 140; Grove, V. C., 478.

Hadamard, J., 477; Hallett, G. H., Jr., 138; Hamel, G., 331; Hart, W. L., 429; Haskins, C. N., 139, 189; Heath, R. S., 93; Hecke, E., 42; Hedrick, E. R., 283; Heegaard, P., 42; Hemke, P. E., 284; Henrici, O., 141; Hildebrandt, T. H., 93; Hill, P. W., 479; Hölder, O., 380; Hollcroft, T. R., 43, 93, 189; Hollcroft, Mrs. T. R., 189; Howe, A. M., 138; Huntington, E. V., 43; Hurwitz, W. A., 237; Hutchinson, C. A., 189.

Jackson, D., 139, 139, 429; James, G., 138; Jensen, J. L. W. V., 476; Jentzch, R., 238; Julia, G., 237. 
Kellogg, O. D., 93, 428, 477; Kempner, A. J., 44; Keppel, H. G., 142, 189; Kiernan, A., 429; King, W. J., 429; Kingston, H. R., 283; Kinney, J. M., 138; Kircher, E. A. T., 478; Kline, J. R., 94, 478; Königsberger, L., 282.

Lamb, H., 42; Lamond, J. K., 381; Lampe, E., 285; Lane, E. P., 138; Larmor, J., 42, 139; Lasley, J. W., Jr., 44; Lattès, S., 141, 237; Laura, E., 42; Lazzarino, O., 331; Le Stourgeon, F. E., 44; LeviCivita, T., 331; Liapounoff, A. M., 480; Light, G. H., 478; Lindsey, L., 140; Longley, W. R., 140; Lorentz, H. A., 282; Lovitt, W. V., 44.

McAtee, J. E., 138, 190; MacLean, N. B., 283; MacMillan, W. D., 139, 139; Marcolongo, R., 42; Martin, A., 142; Mason, M., 93; Mathews, R. M., 284; Matthies, W., 380; Medici, S., 333; Mendenhall, W. O., 189; Mikesh, J. S., 237; Milhaud, G., 284; Miller, F. E., 381; Miller, G. A., 43; Milne, W. E., 237; Mitchell, H. H., 139, 139; Montel, P., 139; Moore, R. E., 93; Moots, E. E., 479; Morgan, F. M., 189; Morley, F., 477; Morris, F. R., 138, 141; Morse, H. C. M., 479; Moulton, F. R., 137, 139, 474; Mullins, G. W., 381; Murnaghan, F. D., 140; Musselman, J. R., 139.

Nassau, J. J., 139; Naylor, Mrs. D., 140; Nelson, A. B., 480; Nelson, C. A., 478; Neovius, E. R., 45; Nicholson, J. L., 380; Noether, F., 332; Noether, M., 282; Nowlan, F. S., 44, 283.

Ott, W. P., 138; Owens, H. B., 283.

Paine, C. P., 138; Parsons, C., 380; Pascal, E., 477; Pfeiffer, G. A., 284; Phalen, H. R., 141; Picard, E., 477; Pizzetti, P., 94; Powell, H. W., 142; Prandtl, L., 476; Pupin, M., 478.

Rambler, O. J., 138; Ramsey, A., 284; Reed, F. W., 237; Rhoton, A. L., 381; Rice, J. N., 139; Richardson, C. H., 140; Richardson, R. G. D., 280; Rider, P. R., 43; Rietz, H. L., 44, 280; Ritt, J. F., 139; Roe, E. D., Jr., 43, 479; Roe, J. R., 138; Roever, W. H., 139; Rosenbach, J. B., 44; Rost, G., 380; Rothrock, D. A., 283; Rouse, L. J., 138; Rowe, J. E., 43, Rupp, C. A., 428.

von Sanden, H., 332; von Schaewen, P., 285; Schick, M. J., 429; Schlink, W., 332; Scott, G. H., 140; Searle, G. M., 45; Seely, C. E., 43, 139; Severini, C., 331; Sharpe, F. R., 478; Shaw, J. B., 44; Shively, L. S., 139; Shoemaker, H. M., 138; Showman, H. M., 479; Silverman, L. L., 141; Simon, M., 45; Simon, W. G., 139, 141; Simpson, T. M., 189; Sisam, C. H., 44; Skinner, A. N., 285; Slaught, H. E., 237, 280; Slobin, H. L., 284, 479; Smith, C. M., 237; Smith, D. E., 43; Smith, E. S., 44; Smith, G. W., 141; Smith, H. L., 139, 283, 479; Smith, M. G., 139, 284; Stark, M. E., 480; Staude, O., 282; Stearns, C. L., 284; Steinitz, 332; Stetson, J. M., 478; Stone, W. B., 238; Sy, F., 139; Sylow, L. M., 284; Sznyther, M. A., 139. 429.

Taylor, E., 478; Taylor, J. S., 139, 479; Tucker, R. S., 283; Tyler, J.,

Underhill, A. L., 479.

Van der Vries, J. N., 332; Vandiver, H. S., 139; Van Vleck, E. B., 477; Veblen, O., 139, 424; Volta, L., 331; Volterra, V., 477.

Wahlin, G. E., 44; Wallenberg, G., 380; Walton, T. O., 141; Warren, L. A. H., 283; Watkeys, C. W., 141; Watson, G. N., 93, 477; Wear, L. E., 93; Weaver, W., 479; Webster, A. G., 43, 282; Weinstein, M. B., 480; Weitzenböck, R., 332; Weyl, H., 42; White, M. B., 480; 
Wiener, N., 43, 479; Wilczynski, E. J., 425; Wilder, C. E., 93, 381, 429; Wilkinson, A. C. L., 425; Wilson, E. B., 425; Winger, R. M., 93; Wolf, C., 141; Wolfe, H. E., 93.

Yaeger, L. E., 140; Yeaton, C. H., 93, 284; Young, J. M., 284; Young, J. W., 189.

Zeldin, S. D., 189; Zeuthen, H. G., 381, 428.

Prizes:

Ackermann-Teubner, 476; Adams, 380; Berlin University, 281; Danish Academy, 476; Italian Society of Sciences, Medal, 331; Paris Academy of Sciences, 42, 139, 237; Prince Jablonowski Society, 281; Royal Society of London, Copley Medal, 282.

Universities and Technical Schools.

Berlin, 281.

Bologna, 40.

California, 475

Catania, 40.

Chicago, 330, 427.

Clark, 43.

Collège de France, 281.

Columbia, 330, 425 .

Cornell, 330, 425.

Genoa, 40.

Harvard, 188, 426.

Illinois, 427.

Johns Hopkins, 475.

Messina, 40.

Naples, 40.

Padua, 41.

Palermo, 41.

Paris, 39.

Pavia, 41.

Pennsylvania, 428.

Pisa, 41 .

Princeton, 426.

Rome, 41 .

Strasbourg, 282, 476.

Turin, 42 .

Yale, 428. 